\title{
Occurrence and trophic transfer of per- and polyfluoroalkyl substances in an Antarctic ecosystem
}

\author{
$\mathrm{Ke} \mathrm{GaO}^{1}$, HuiJuan Li ${ }^{1}$, JiAnJie Fu ${ }^{1,{ }^{*}}$, AiQIAN ZhanG ${ }^{1 *}$, \\ GUIBIN JIANG ${ }^{1}$ \\ ${ }^{1}$ State Key Laboratory of Environmental Chemistry and \\ Ecotoxicology, Research Center for Eco-Environmental \\ Sciences, Chinese Academy of Sciences, Beijing, China, \\ iifu@rcees.ac.cn; aqzhang@rcees.ac.cn.A
}

Per- and polyfluoroalkyl substances (PFASs) are a class of anthropogenic compounds that have broad industrial and commercial applications, including aqueous firefighting foam, floor polishes, insecticides, textiles, carpets, and food packaging. Information on the occurrence and trophodynamics of per- and polyfluoroalkyl substances (PFASs) in the Antarctic region is limited. In this study, we systematically collected sediment, algae, and animal samples from the Fildes Peninsula at King George Island and Ardley Island, Antarctica, and aimed to investigate the occurrence and profiles of PFASs in Antarctic ecosystems. Moreover, the trophodynamic behaviors of PFASs were evaluated. To our knowledge, this is the first study on the trophic transfer of PFASs in the Antarctic food chain, and our present study provides valuable information on the environmental and biological behaviors of PFASs in the Antarctic region.

$\sum$ PFASs ranged from $0.50 \pm 38.0 \mathrm{ng} / \mathrm{g} \mathrm{dw}$ (dry weight) in algae to $4.97 \pm 1.17 \mathrm{ng} / \mathrm{g} \mathrm{dw}$ in Neogastropoda (Ngas), which was lower than those in the low- and mid-latitude regions and even Arctic regions. Perfluorobutyric acid (PFBA) was predominant with detection frequencies above $50 \%$ in all types of samples, and the relative contribution of PFBA ranged from $22 \%$ to $57 \%$ in the biota samples. The biomagnification factors of PFBA, perfluoroheptanoate (PFHpA), perfluorohexane sulfonate (PFHxS), and perfluorooctane sulfonate (PFOS) between Archaeogastropoda (Agas) and Ngas were $0.67 \pm 0.54$, $0.77 \pm 0.38, \quad 1.04 \pm 1.56, \quad 3.30 \pm 4.07, \quad$ and $1.61 \pm 0.89$, respectively. The trophic magnification factors of PFHxS and PFOS were 2.09 and 2.92, respectively, which indicated that they could be biomagnified through the food chain. Considering the increasing production and uncertain toxicological risks of emerging PFASs and the sensitive ecosystems in Antarctic regions, more attention should be paid, especially for the short-chain ones in the Antarctic region.

Acknowledgements:The authors greatly thank the Chinese Arctic and Antarctic Administration for the arrangement of the Chinese Antarctic Scientific Expedition. 